\title{
Determinación de la función fotocatalítica de recubrimientos sobre soporte cerámico
}

\author{
M. C. BORDES, A. MORENO, E. BOU, V. SANZ \\ Instituto de Tecnología Cerámica. Asociación de Investigación de las Industrias Cerámicas \\ Universitat Jaume I. Castellón. España
}

\begin{abstract}
La fotocatálisis es un proceso en el que la incidencia de radiación ultravioleta sobre un semiconductor produce la degradación de la materia orgánica en contacto con el mismo. Este proceso encuentra diversas aplicaciones que requieren el uso del fotocatalizador (semiconductor) en suspensión o soportado sobre un sustrato.

La aplicación de recubrimientos fotocatalíticos sobre sustratos cerámicos da lugar a la obtención de superficies multifuncionales con propiedades autolimpiantes relacionadas con la capacidad de degradación e incluso mineralización de compuestos orgánicos presentes en el agua o el aire.

La preparación de los recubrimientos soportados sobre el sustrato cerámico se realiza mediante la aplicación de suspensiones con un tamaño de partícula nanométrico $\left(10^{-9} \mathrm{~m}\right)$. Debido a su pequeño tamaño de partícula este tipo de materiales presentan propiedades distintas a las de los materiales macroscópicos.

La reciente aparición de materiales con actividad fotocatalítica hace necesaria la existencia de un método para conocer y cuantificar dicha propiedad. Actualmente no existe ninguna norma para la determinación de la función fotocatalítica de materiales. Por ello en el presente trabajo, se ha llevado a cabo la puesta a punto de un método de ensayo que permita la determinación de dicha función para materiales soportados sobre un sustrato cerámico.
\end{abstract}

Palabras clave: Recubrimientos, cerámica, función fotocatalítica, $\mathrm{TiO}_{2}$

Determination of the photocatalytic function of coatings on ceramic substrates

Photocatalysis is a process in which the impingement of UV radiation on a semiconductor causes the organic matter in contact with the semiconductor to degrade. The process is found in various applications that require the use of the photocatalyst (semiconductor) in suspension or supported on a substrate.

The application of photocatalytic coatings onto ceramic substrates yields multifunctional surfaces with self-cleaning properties related to their capacity to degrade, and even mineralise, organic compounds present in water or air.

The photocatalytic coatings supported on ceramic substrates are obtained by applying suspensions of nanometre-sized particles $\left(10^{-9} \mathrm{~m}\right)$ onto the substrate. Owing to the small particle size, this type of material has different properties from those of macroscopic materials.

The recent appearance of photocatalytically active materials requires having a method for determining and quantifying photocatalytic activity. No standard is currently available for determining the photocatalytic function of materials. The present study was undertaken, therefore, to fine-tune a test method that would allow determination of the photocatalytic function in materials supported on a ceramic substrate

Keywords: Coatings, ceramics, photocatalytic function, $\mathrm{TiO}_{2}$

\section{1- 2. INTRODUCCIÓN}

La fotocatálisis es un proceso en el que la incidencia de radiación ultravioleta sobre un semiconductor produce la degradación de la materia orgánica en contacto con el mismo.

El proceso fotocatalítico comienza con la generación de pares electrón/hueco en las partículas del semiconductor por efecto de la luz $(1,2)$. Así, cuando sobre el semiconductor incide un fotón con una energía hv igual o superior al salto de banda del mismo, se produce el salto de un electrón (e-) de la banda de valencia a la de conducción, generándose un hueco $\left(\mathrm{h}^{+}\right)$en la primera. Los electrones que llegan a la banda de conducción pueden desplazarse a través de la red del semiconductor y del mismo modo pueden hacerlo los huecos generados en la banda de valencia.

Los electrones y los huecos generados pueden migrar a la superficie del semiconductor llevando a cabo una transferencia electrónica con las sustancias adsorbidas en la superficie de la partícula. Por una parte, los electrones reducen a un aceptor de electrones (normalmente oxígeno), y por otra, los huecos pueden aceptar un electrón de una especie dadora de electrones, de manera que esta especie se oxide, según las reacciones: 
$\mathrm{e}^{-}+\mathrm{A} \rightarrow \mathrm{A}^{-}$
$\mathrm{h}^{+}+\mathrm{D} \rightarrow \mathrm{D}$

Por tanto, la captura de un electrón por la especie A da lugar a un radical $\mathrm{A}^{-}$, mientras que la interacción entre la especie D y el hueco da lugar a un radical $\mathrm{D}^{+}$. Estos radicales son muy reactivos y pueden reaccionar entre ellos y con otras especies adsorbidas o bien difundirse desde la superficie del semiconductor y participar en una reacción química en el seno de la fase acuosa, en el caso de que aquel se encuentre en contacto con una disolución.

Existen diversos materiales semiconductores que pueden ser utilizados como fotocatalizadores ya que poseen una diferencia de energía entre la banda de valencia y la de conducción, denominada potencial de banda, adecuada para que se produzcan las reacciones fotocatalíticas (reducción del oxígeno y oxidación del agua adsorbida). Sin embargo, el $\mathrm{TiO}_{2}$ debido a su estabilidad en disolución acuosa y no toxicidad es el más ampliamente utilizado (3).

Los recubrimientos fotocatalíticos estudiados en el presente trabajo contienen como catalizador el semiconductor dióxido de titanio $\left(\mathrm{TiO}_{2}\right)$. La secuencia de reacciones que tienen lugar en la superficie del $\mathrm{TiO}_{2}$ fotocatalítico en presencia de oxígeno y agua, son (4):

(1) $\mathrm{TiO}_{2}+\mathrm{h} v \leftrightarrow \mathrm{h}_{\text {b.v. }}^{+}+\mathrm{e}_{\text {b.c. }}^{-}$

(2) $\mathrm{e}_{\text {b.c. }}^{-}+\mathrm{O}_{2} \rightarrow \cdot \mathrm{O}_{2}$

(3) $\mathrm{h}_{\text {b. } .}^{+}+\mathrm{H}_{2} \mathrm{O}_{\text {ads }} \leftrightarrow \mathrm{H}^{+}{ }_{\text {ads }}+\cdot \mathrm{OH}_{\text {ads }}$

Los huecos $\mathrm{h}_{\text {b.v. }}^{+} \mathrm{y}$ los radicales ${ }^{\bullet} \mathrm{OH}$ generados producen la oxidación y la degradación de las sustancias orgánicas presentes:

(4) $\mathrm{R}+\mathrm{OH} \rightarrow \mathrm{R}^{\prime}+\mathrm{H}_{2} \mathrm{O}$

(5) $\mathrm{R}+\mathrm{h}_{\mathrm{BV}}^{+} \rightarrow \mathrm{R}^{+\cdot}{ }^{\circledR}$ productos de reacción

La reciente aparición de materiales con actividad fotocatalítica que encuentran diversas aplicaciones $(5,6)$, hace necesaria la existencia de un método para conocer y cuantificar dicha propiedad. Actualmente no existe ninguna norma para la determinación de la función fotocatalítica de materiales ya sea soportados sobre un sustrato o en polvo. Por ello en este trabajo se ha llevado a cabo la puesta a punto de un método de ensayo que permita la determinación de dicha función para materiales soportados sobre un sustrato cerámico.

La fotocatálisis es un proceso en el que la incidencia de radiación ultravioleta sobre un semiconductor produce la degradación de la materia orgánica en contacto con el mismo. Por ello, la función fotocatalítica de un material se puede determinar siguiendo la variación que experimenta la concentración de una sustancia orgánica en disolución acuosa en contacto con el fotocatalizador y estando expuesta a la radiación ultravioleta.

El compuesto orgánico utilizado en el presente trabajo para la determinación de la función fotocatalítica de los recubrimientos es azul de metileno, éste es un compuesto utilizado habitualmente en este tipo de determinaciones $(7,8$, $9,10,11,12)$ que absorbe la radiación visible y origina un color azul en disolución acuosa. Esta propiedad permite el uso de la espectroscopia ultravioleta-visible para la determinación de la concentración del azul de metileno en disolución acuosa.

Sin embargo, hay que tomar en consideración que la exposición del azul de metileno a la radiación ultravioleta produce además su degradación por fotólisis, es decir, la absorción de la radiación aumenta el contenido energético de la molécula que pasa a un estado inestable. La energía absorbida se disipa en forma de reacciones químicas por radicales libres que degradan el compuesto. Debido a ello, la exposición a la radiación debe realizarse sobre la disolución de azul de metileno en contacto con el fotocatalizador y en ausencia del mismo para determinar así la influencia de la fotólisis en la disminución de la concentración del compuesto.

\section{EXPERIMENTAL}

\subsection{Materiales}

Las disoluciones de azul de metileno se prepararon utilizando azul de metileno con una riqueza del $82 \%$ disolviendo la cantidad necesaria en agua destilada para obtener la concentración a ensayar.

Para la obtención de las superficies cerámicas con propiedades fotocatalíticas, se utilizó un producto comercial consistente en un sistema de recubrimientos de doble capa. Este sistema comprende la aplicación sucesiva de dos suspensiones, siendo la primera de ellas una suspensión coloidal de $\mathrm{SiO}_{2}$ y la segunda una suspensión de nanopartículas de $\mathrm{TiO}_{2}$

La puesta a punto del ensayo se realizó sobre distintos tipos de superficies cerámicas. El recubrimiento fotocatalítico se aplicó sobre los sustratos más representativos en el sector de baldosas cerámicas, incluyendo siete superficies con distintas morfologías.

\subsection{Procedimiento experimental}

\subsubsection{Caracterización de las superficies cerámicas}

Previamente a la aplicación del recubrimiento fotocatalítico, se llevó cabo la caracterización de las superficies cerámicas determinándoles la rugosidad superficial.

La rugosidad se determinó con un rugosímetro utilizando un palpador de punta de diamante de curvatura $90^{\circ}$ y radio $5 \mu \mathrm{m}$. Sobre cada una de las superficies estudiadas se obtuvieron tres perfiles. Para su obtención se utilizó un cut-off de $2.5 \mathrm{~mm}$.

De cada uno de los perfiles obtenidos se calculó el parámetro Ra de rugosidad:

$\mathrm{R}$ : Media aritmética de los valores absolutos de la distancia de los puntos que componen el perfil a una línea media. Es el parámetro más representativo de la rugosidad media de una superficie.

\subsubsection{Obtención de los recubrimientos fotocatalíticos}

La preparación de las superficies cerámicas recubiertas se realizó utilizando un producto comercial que comprende la aplicación sucesiva de dos suspensiones, siendo la primera de ellas una suspensión coloidal de $\mathrm{SiO}_{2}$ y la segunda una suspensión de nanopartículas de $\mathrm{TiO}_{2}$.

La aplicación de las suspensiones se llevó a cabo mediante un sistema de pulverización con generación de microgotas que permite aplicar el gramaje necesario para obtener una capa homogénea con unos pocos micrómetros de espesor. 
En primer lugar se realizó la aplicación de la suspensión de $\mathrm{SiO}_{2}$ y, una vez seca la capa obtenida, se aplicó la suspensión de $\mathrm{TiO}_{2}$ sobre los distintos soportes cerámicos.

Una vez aplicadas las probetas se sometieron a un tratamiento térmico en un horno eléctrico de laboratorio a una temperatura de $400^{\circ} \mathrm{C}$, para lograr el anclaje del fotocatalizador al sustrato y la eliminación de los aditivos y disolventes de la suspensión.

\subsubsection{Determinación de la concentración de azul de} metileno

La determinación de la absorbancia de las disoluciones de azul de metileno se realizó utilizando un espectrómetro UVVisible. Las medidas se realizaron a una longitud de onda de $665 \mathrm{~nm}$ correspondiente al pico de máxima absorción del azul de metileno.

Previamente a la determinación de la concentración de las disoluciones se obtuvo la recta de calibrado del espectrómetro que relaciona directamente la absorbancia con la concentración de la muestra.

En aquellos casos en los que la concentración era superior a 5 ppm, para determinar su absorbancia, se realizó la dilución de la muestra necesaria para obtener una concentración en el intervalo entre 0 y 5 ppm entre las cuales la relación entre la absorbancia y la concentración era lineal.

\subsubsection{Exposición a la radiación UV}

La exposición de la disolución de azul de metileno a la radiación se realizó utilizando un montaje experimental que permitía mantener la disolución sobre la superficie a ensayar en contacto con oxígeno del ambiente sin que existieran pérdidas de líquido. El volumen de disolución utilizado en los ensayos fue de $1 \mathrm{ml}$ por $\mathrm{cm}^{2}$ de superficie a ensayar.

La exposición a la radiación se realizó en una cámara de insolación compuesta de una lámpara de arco de vapor de mercurio a presión media que emite luz ultravioleta con tres potencias distintas: 400, 800 y $1200 \mathrm{~W}$. Los ensayos se realizaron situando el montaje con la disolución bajo la lámpara a una distancia de $65 \mathrm{~cm}$ de la misma.

\subsubsection{Determinación de la función fotocatalítica}

La determinación de la función fotocatalítica del recubrimiento, se realizó utilizando una baldosa con recubrimiento y otra sin él para determinar así la influencia de la fotólisis en la disminución de la concentración del compuesto.

Con el fin de permitir la adsorción del azul de metileno sobre la superficie, previamente a la exposición a la radiación, las probetas se mantuvieron en contacto con la disolución 30 minutos en la oscuridad utilizando el montaje experimental indicado con anterioridad y determinándose la concentración transcurrido este tiempo. A continuación se realizó la exposición a la radiación ultravioleta, extrayéndose una muestra de las disoluciones tras cada exposición para la determinación de la concentración. Una vez determinada la concentración, la muestra extraída se volvía a introducir en el montaje y continuaba la exposición hasta obtener una concentración inferior al 15\% de la inicial.

Los ensayos se realizaron a una temperatura de $30^{\circ} \mathrm{C}$ no existiendo variaciones apreciables de la misma durante la realización de los mismos.

\section{RESULTADOS}

La puesta a punto de un método para determinar una variable característica de un material hace necesario establecer unas condiciones de ensayo estándar que permitan obtener una repetitividad. Para ello se han estudiado las variables relacionadas con los tres factores que intervienen en la realización del ensayo: la fuente de radiación ultravioleta, la disolución de azul de metileno y la superficie cerámica.

\subsection{Influencia de las características de la fuente de radiación ultravioleta}

Se ha estudiado la influencia de la fuente de radiación ultravioleta utilizada en el ensayo sobre la concentración de azul de metileno (en adelante AM) en la disolución variando la potencia de la lámpara instalada en la cámara de insolación. Las potencias estudiadas han sido: $400 \mathrm{~W}, 800 \mathrm{~W}$ y $1200 \mathrm{~W}$.

Los ensayos se realizaron sobre un soporte cerámico no esmaltado con una concentración de AM inicial de 10 ppm. Cada una de las determinaciones se llevó a cabo sobre una probeta con recubrimiento fotocatalítico en paralelo con otra probeta sin recubrimiento.

Las probetas con la disolución de AM, se expusieron a la radiación ultravioleta durante distintos tiempos hasta alcanzar una concentración inferior al 15\% de la inicial para cada una de las tres potencias estudiadas. Tras cada uno de los tiempos de insolación se extrajeron las disoluciones y se determinó su absorbancia, obteniéndose a partir de la recta de calibrado la concentración de AM en cada una de las muestras.

En la figura 1 se muestra la variación de concentración que experimentan las disoluciones de $\mathrm{AM}$ en contacto con las probetas con recubrimiento y sin él con el tiempo, para la potencia de $400 \mathrm{~W}$.

Existen diferencias claras entre la concentración de las disoluciones para cada uno de los tiempos de exposición a la

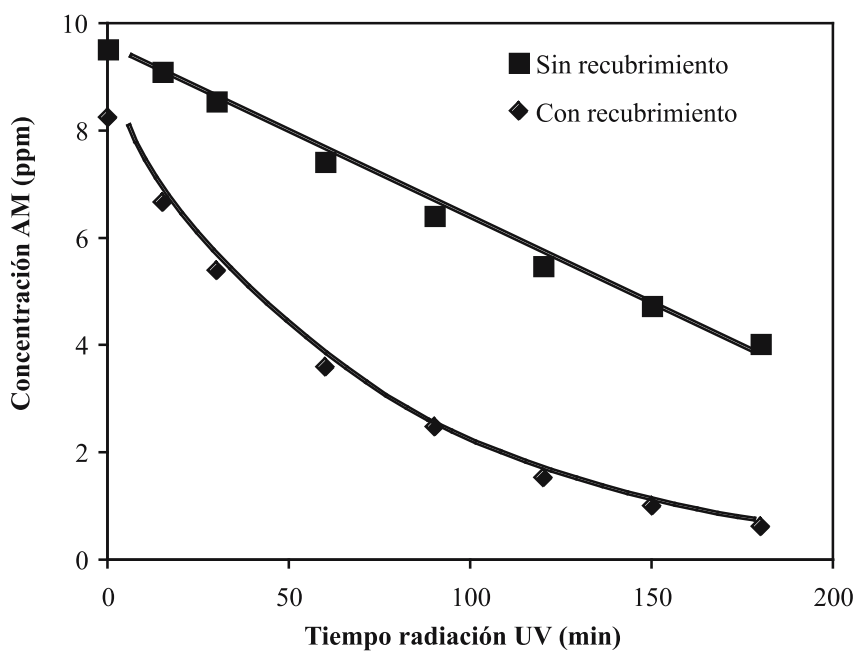

Fig. 1- Variación de la concentración de Azul de Metileno con el tiempo de exposición a la radiación ultravioleta para una potencia de $400 \mathrm{~W}$.

radiación. Así se obtiene una disminución de concentración mucho más acusada para la muestra con recubrimiento. De este modo, la degradación de AM debida a los procesos 
conjuntos de fotólisis y fotocatálisis que se produce en la probeta con recubrimiento, es mayor que la degradación obtenida sólo por fotólisis que es la que se produce en la probeta sin recubrimiento. Por tanto, se comprueba que el recubrimiento aplicado sobre la superficie ensayada presenta actividad fotocatalítica.

La concentración de las disoluciones en contacto con las dos superficies, tras el tiempo de permanencia en la oscuridad es inferior a 10 ppm, por tanto existe además una adsorción inicial de AM sobre la superficie de las probetas, siendo ésta mayor en el caso de la probeta con recubrimiento.

La cinética del proceso de degradación es distinta para las disoluciones en contacto con las probetas con recubrimiento y sin él. La degradación en la probeta sin recubrimiento presenta una variación lineal de la concentración con el tiempo lo que indica que sigue una cinética de orden cero que se puede ajustar a una ecuación del tipo:

$$
\mathrm{C}=\mathrm{C}_{0}+\mathrm{k} \cdot \mathrm{t}
$$

donde $\mathrm{C}_{0}$ es la concentración inicial (tras el tiempo de permanencia en la oscuridad) y $\mathrm{k}$ es la constante de velocidad.

En el caso de la disolución en contacto con el recubrimiento la variación de la concentración del compuesto con el tiempo se obtiene mediante la ecuación de Langmuir-Hinshelwood $(8,9,10,11,12,13)$, que para concentraciones bajas es:

$$
-\frac{d C}{d t}=k^{\prime} C
$$

correspondiente a una cinética de pseudoprimer orden cuya integración da lugar a:

$$
\ln \frac{C_{0}}{C}=k^{\prime} t
$$

La representación de $\ln \left(\mathrm{C}_{0} / \mathrm{C}\right)$ frente al tiempo da lugar a una recta cuya pendiente es la constante de velocidad $\mathrm{k}^{\prime}$. En la figura 2 se muestra la variación del $\ln \left(\mathrm{C}_{0} / \mathrm{C}\right)$ con el tiempo de exposición a la radiación para la baldosa con recubrimiento.

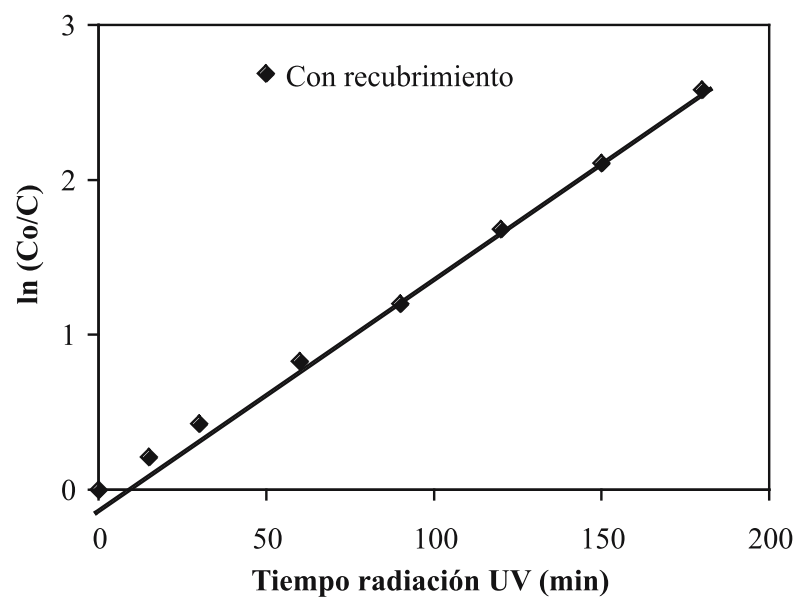

Fig. 2- Variación del $\ln \left(\mathrm{C}_{0} / \mathrm{C}\right)$ con el tiempo de exposición a la radiación ultravioleta para la baldosa con recubrimiento para una potencia de $400 \mathrm{~W}$.
Para las potencias de la lámpara de 800 y 1200 W se obtienen curvas de degradación similares a las obtenidas a 400W. De este modo se obtienen diferencias entre las concentraciones de las disoluciones de AM de las probetas con recubrimiento y sin él para cada uno de los tiempos de exposición ensayados. Sin embargo en los dos casos estas diferencias son menores que las obtenidas para la potencia de $400 \mathrm{~W}$.

Además para estas dos potencias se observa que las cinéticas del proceso de degradación para las disoluciones en contacto con las probetas con recubrimiento y sin él, son del mismo orden que las obtenidas para la potencia de $400 \mathrm{~W}$, y se ajustan a las ecuaciones indicadas anteriormente.

En la tabla I se indican los parámetros obtenidos del ajuste de la variación de concentración con el tiempo a las ecuaciones cinéticas (1) y (3) para las probetas con y sin recubrimiento a las tres potencias ensayadas.

TABLA I. PARÁMETROS DEL AJUSTE DE LA VARIACIÓN DE CONCENTRACIÓN CON EL TIEMPO A LA ECUACIONES CINÉTICAS (1) Y (3) PARA LAS DISOLUCIONES DE AM DE LAS PROBETAS CON Y SIN RECUBRIMIENTO A LAS POTENCIAS DE 400, $800 \mathrm{Y}$ $1200 \mathrm{~W}$.

\begin{tabular}{|c|c|c|c|c|c|}
\hline \multirow{2}{*}{$\begin{array}{c}\text { Potencia } \\
(\mathrm{W})\end{array}$} & \multicolumn{3}{|c|}{ Probetas sin recubrimiento } & \multicolumn{2}{c|}{$\begin{array}{c}\text { Probetas con } \\
\text { recubrimiento }\end{array}$} \\
\cline { 2 - 6 } & $\begin{array}{c}\mathrm{C}_{0} \\
(\mathrm{ppm})\end{array}$ & $\begin{array}{c}\mathrm{k} \cdot 10^{2} \\
(\mathrm{ppm} / \mathrm{min})\end{array}$ & $\mathrm{r}^{2}$ & $\begin{array}{c}\mathrm{k}^{\prime} \cdot 10^{2} \\
\left(\mathrm{~min}^{-1}\right)\end{array}$ & $\mathrm{r}^{2}$ \\
\hline 400 & 9,4 & 3,1 & 0,994 & 1,4 & 0,999 \\
\hline 800 & 9,0 & 8,1 & 0,997 & 2,9 & 0,997 \\
\hline 1200 & 9,0 & 11,7 & 0,990 & 4,5 & 0,995 \\
\hline
\end{tabular}

Se observa que para las potencias y los dos tipos de probetas ensayadas se obtienen variaciones de concentración con el tiempo que se ajustan a las ecuaciones cinéticas propuestas puesto que en todas ellas el coeficiente de regresión lineal $\left(\mathrm{r}^{2}\right)$, indicativo de la bondad del ajuste, es próximo a 1.

Por tanto, la degradación de AM que se produce en las probetas sin recubrimiento que es debida a la fotólisis sigue una cinética de orden cero en la que la concentración varía de forma lineal con el tiempo, observándose que la constante de velocidad aumenta con la potencia ensayada.

En el caso de las probetas con recubrimiento la degradación de AM que se produce debida a los procesos de fotólisis y fotocatálisis sigue una cinética de pseudoprimer orden, presentando también un aumento de la constante de velocidad con la potencia ensayada.

A partir del estudio del efecto de la potencia de la lámpara utilizada como fuente de radiación ultravioleta sobre la concentración del AM en disolución, se concluye, que aquella que produce una fotólisis menos acusada y permite por tanto determinar la función fotocatalítica del recubrimiento es de 400 W. Para potencias superiores se produce una degradación muy acusada del compuesto orgánico en la disolución que dificulta la determinación de la actividad fotocatalítica de la superficie.

\subsection{Influencia de las características de la disolución de azul de metileno}

Una vez estudiadas las condiciones de radiación de la muestra que permiten la determinación de la función fotocatalítica de los recubrimientos, es necesario estudiar la influencia de las variables correspondientes a la disolución tomada como fuente de materia orgánica en el ensayo.

Las variables de ensayo que hacen referencia a la disolución son el volumen y la concentración inicial de AM, ambas 
determinan el número de moles que deben ser degradados por el recubrimiento.

Puesto que se está ensayando un proceso en el que se produce una reacción superficial, es necesario mantener una relación entre el volumen de disolución y la superficie en contacto con la misma constante. Así se fijó este parámetro en $1 \mathrm{ml} / \mathrm{cm}^{2}$ y se tomó como variable la concentración inicial de la disolución de AM.

Se ensayaron tres concentraciones de AM en la disolución inicial: 5, 10 y $20 \mathrm{ppm}$. En la tabla II se indica los moles por $\mathrm{m}^{2}$ de superficie correspondientes a cada concentración.

TABLA II. CONCENTRACIÓN (PPM) DE LAS DISOLUCIONES DE AM Y MOLES INICIALES POR $\mathrm{M}^{2}$ DE SUPERFICIE ENSAYADOS PARA CADA UNA DE ELLAS.

\begin{tabular}{|c|c|}
\hline Concentración $(\mathrm{ppm})$ & Moles AM $/ \mathrm{m}^{2} .10^{4}$ \\
\hline 5 & 1,6 \\
\hline 10 & 3,1 \\
\hline 20 & 6,3 \\
\hline
\end{tabular}
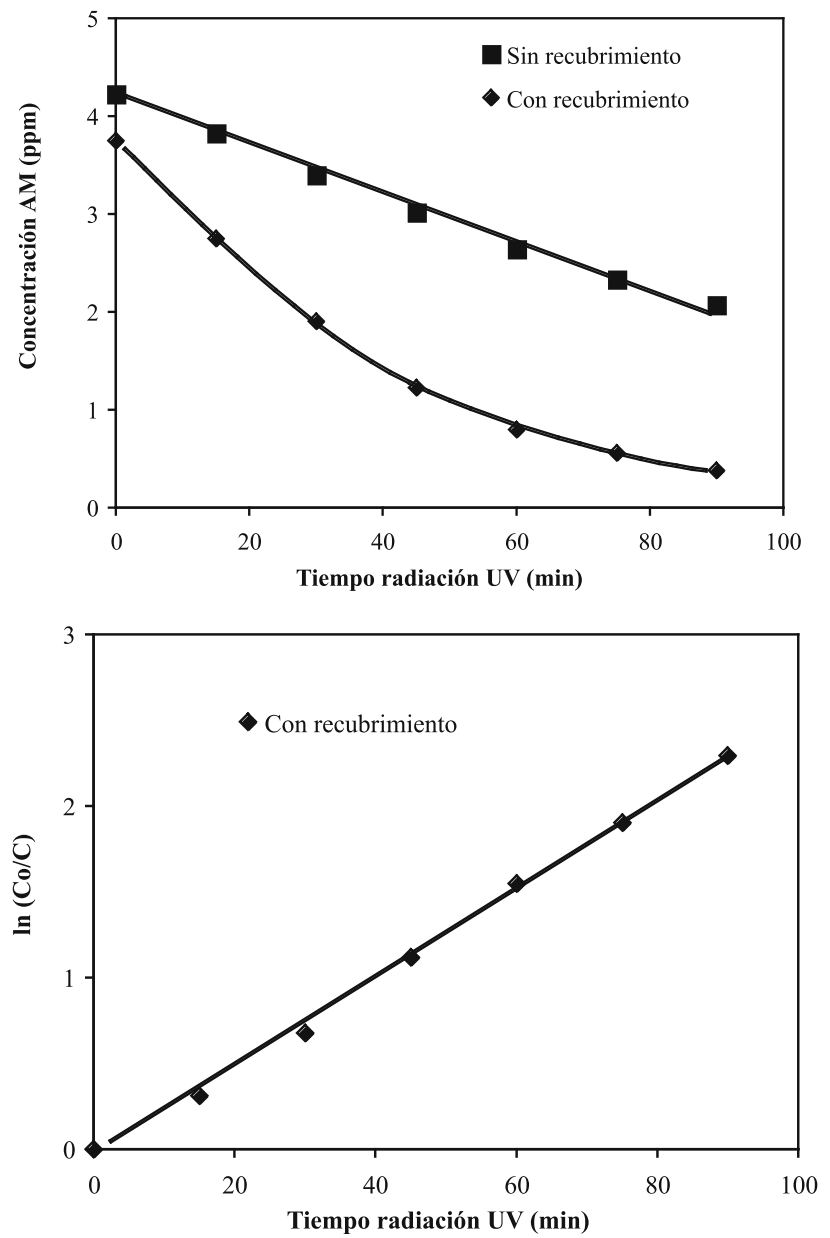

Fig. 3- Variación de la concentración de Azul de Metileno y del $\ln \left(\mathrm{C}_{0}\right)$ C) con el tiempo de exposición a la radiación ultravioleta para una concentración de 5 ppm.

TABLA III. PARÁMETROS DEL AJUSTE DE LA VARIACIÓN DE CONCENTRACIÓN CON EL TIEMPO A LA ECUACIONES CINÉTICAS (1) Y (3) PARA LAS DISOLUCIONES DE AM DE LAS PROBETAS CON Y SIN RECUBRIMIENTO PARA LAS CONCENTRACIONES INICIALES DE 5, 10 Y 20 PPM.
Los ensayos se realizaron en la cámara de insolación con una intensidad de radiación de $400 \mathrm{~W}$ y sobre el soporte cerámico no esmaltado utilizado anteriormente. Cada una de las determinaciones se llevó a cabo sobre una probeta con recubrimiento fotocatalítico en paralelo con otra probeta sin recubrimiento.

El método utilizado para la determinación de la variación de concentración con el tiempo de exposición a la radiación fue el mismo que el indicado en el apartado anterior, permaneciendo previamente las probetas en contacto con la disolución de AM durante 30 minutos en la oscuridad.

En la figura 3 se muestra la variación de concentración que experimentan las disoluciones de AM en contacto con las probetas con recubrimiento y sin él con el tiempo para la concentración inicial de $5 \mathrm{ppm}$. En la misma figura se muestra la variación del $\ln \left(\mathrm{C}_{0} / \mathrm{C}\right)$ con el tiempo de exposición a la radiación para la baldosa con recubrimiento.

Para las tres concentraciones ensayadas se obtiene una disminución de la concentración de AM con el tiempo de exposición a la radiación, siendo ésta más acusada en el caso de las probetas con recubrimiento. Así en todos los casos se observa el efecto fotocatalítico del recubrimiento que produce una mayor degradación del AM en la disolución.

En la tabla III se indican los parámetros obtenidos del ajuste de la variación de concentración con el tiempo a las ecuaciones cinéticas (1) y (3) de las probetas con y sin recubrimiento para las tres concentraciones ensayadas.

Se observa que para las concentraciones iniciales y los dos tipos de probetas ensayadas se obtienen variaciones de concentración con el tiempo que se ajustan a las ecuaciones cinéticas propuestas puesto que en todas ellas el coeficiente de regresión lineal $\left(\mathrm{r}^{2}\right)$ es próximo a 1.

Las constantes de velocidad siguen tendencias distintas al aumentar la concentración inicial de AM. Así en ausencia de recubrimiento, cuando aumenta la concentración inicial también lo hace la constante de velocidad k. Mientras en las probetas con recubrimiento cuando aumenta la concentración disminuye la constante $\mathrm{k}^{\prime}$.

La concentración que permite diferenciar con mayor claridad la degradación que se produce en las probetas con y sin recubrimiento es la de $10 \mathrm{ppm}$. Para concentraciones inferiores la degradación en ambos tipos de probetas se produce en un tiempo muy corto y los valores son muy bajos. Mientras que para $20 \mathrm{ppm}$, como consecuencia de la baja constante de velocidad que se obtiene para la degradación en la probeta con recubrimiento, es necesario utilizar tiempos de exposición mayores para degradar el compuesto, siendo menos acusadas las diferencias de concentración entre las disoluciones correspondientes a ambos tipos de superficie.

\subsection{Influencia de las características de la superficie cerámica}

La fotocatálisis es un proceso en el que las reacciones tienen lugar en la superficie del sustrato. Para ello es necesario que se produzca el anclaje del $\mathrm{TiO}_{2}$ formando una capa de unos pocos micrómetros de espesor lo más homogénea

\begin{tabular}{|c|c|c|c|c|c|}
\hline \multirow{2}{*}{$\begin{array}{c}\text { Concentración inicial } \\
(\mathrm{ppm})\end{array}$} & \multicolumn{3}{|c|}{ Probetas sin recubrimiento } & \multicolumn{2}{|c|}{ Probetas con recubrimiento } \\
\hline & (ppm) & $\mathrm{k} .10^{2}(\mathrm{ppm} / \mathrm{min})$ & $\mathrm{r}^{2}$ & $\left(\min ^{-1}\right)$ & $\mathrm{r}^{2}$ \\
\hline 5 & 4,2 & 2,4 & 0,995 & 2,5 & 0,997 \\
\hline 10 & 9,4 & 3,1 & 0,994 & 1,4 & 0,999 \\
\hline 20 & 19,9 & 3,7 & 0,991 & 0,5 & 0,994 \\
\hline
\end{tabular}


TABLA IV. RUGOSIDAD DE LOS SOPORTES CERÁMICOS CARACTERIZADOS.

\begin{tabular}{|c|c|}
\hline Soporte & Ra $(\mu \mathrm{m})$ \\
\hline A & 0.06 \\
\hline B & 0.14 \\
\hline C & 0.91 \\
\hline D & 1.26 \\
\hline E & 2.18 \\
\hline F & 2.79 \\
\hline G & 3.16 \\
\hline
\end{tabular}

posible y la adsorción del azul de metileno sobre la superficie. En los apartados anteriores se ha determinado la función fotocatalítica del recubrimiento utilizando como sustrato una superficie cerámica no esmaltada y se han establecido unas condiciones de ensayo estándar. En este apartado se va a determinar dicha función sobre superficies con diferentes características con el fin de estudiar la viabilidad del uso del método de ensayo puesto a punto sobre distintas superficies cerámicas.

El estudio se ha llevado a cabo sobre siete sustratos cerámicos cuya superficie presentaba distinta rugosidad. Previamente a la aplicación del recubrimiento fotocatalítico se realizó una caracterizaron de las superficies cerámicas determinando esta variable para cada una de ellas. En la tabla IV se indican los resultados de dicha caracterización, en la que el soporte $\mathrm{C}$, es el que se había ensayado en los apartados anteriores. en las probetas con recubrimiento. Cada soporte muestra una variación de concentración distinta tanto para la probeta con recubrimiento como sin él; este hecho indica que la fotólisis y la fotocatálisis se producen con distinta intensidad en cada caso, aunque en todos ellos se observa la misma tendencia.

En la tabla V se indican los parámetros obtenidos de los ajustes de la variación de concentración con el tiempo a las ecuaciones cinéticas (1) y (3) para las probetas sin recubrimiento y con él correspondientes a los distintos soportes ensayados.

La degradación que se produce en los dos tipos de probetas para todos los soportes ensayados se ajusta a las ecuaciones cinéticas propuestas $(1$ y 3$)$, como indican los coeficientes de regresión lineal $\left(\mathrm{r}^{2}\right)$ obtenidos.

En el caso de las superficies recubiertas se produce una adsorción inicial de AM mayor que cuando no está presente el recubrimiento, aunque existen diferencias entre los distintos soportes ensayados. Esto puede ser debido a la distinta morfología de las superficies ensayadas.

Las constantes de velocidad obtenidas para las muestras sin recubrimiento correspondientes a los distintos soportes ensayados son del mismo orden de magnitud. Esto mismo se obtiene en los soportes con recubrimiento.

Por tanto, para todos los soportes estudiados, se puede determinar la función fotocatalítica del recubrimiento, mediante la variación de la concentración de azul de metileno con el tiempo y la obtención de la constante de velocidad. En todos ellos para las condiciones ensayadas, también se produce una disminución de concentración debida a la fotólisis del compuesto, aunque ésta menos acusada que la

TABLA V. PARÁMETROS DEL AJUSTE DE LA VARIACIÓN DE CONCENTRACIÓN CON EL TIEMPO A LA ECUACIONES CINÉTICAS (1) Y (3) PARA LAS DISOLUCIONES DE AM DE LAS PROBETAS CON Y SIN RECUBRIMIENTO PARA LOS SOPORTES ENSAYADOS.

\begin{tabular}{|c|c|c|c|c|c|}
\hline \multirow{2}{*}{ Soporte } & \multicolumn{3}{|c|}{ Probetas sin recubrimiento } & \multicolumn{2}{c|}{ Probetas con recubrimiento } \\
\cline { 2 - 6 } & Co $(\mathrm{ppm})$ & $\mathrm{k} \cdot 10^{2}(\mathrm{ppm} / \mathrm{min})$ & $\mathrm{r}^{\prime} \cdot 10^{2}\left(\mathrm{~min}^{-1}\right)$ & $\mathrm{r}^{2}$ \\
\hline A & 9,7 & 3,0 & 0,993 & 1,2 & 0,998 \\
\hline B & 8,4 & 3,3 & 0,990 & 1,4 & 0,995 \\
\hline C & 9,4 & 3,1 & 0,994 & 1,4 & 0,999 \\
\hline D & 9,8 & 2,9 & 0,995 & 1,3 & 0,992 \\
\hline E & 9,7 & 2,9 & 0,994 & 1,3 & 0,993 \\
\hline F & 9,0 & 2.9 & 0,994 & 1,4 & 0,999 \\
\hline G & 9,0 & 3,2 & 0,993 & 1,6 & 0,999 \\
\hline
\end{tabular}

La preparación del recubrimiento fotocatalítico sobre los soportes caracterizados se realizó según el método indicado anteriormente (apartado 2.2.2.).

Los ensayos se realizaron en la cámara de insolación utilizando una potencia de la lámpara de $400 \mathrm{~W}$, introduciendo un volumen $1 \mathrm{ml}$ por $\mathrm{cm}^{2}$ de superficie de disolución de $\mathrm{AM}$ con una concentración de $10 \mathrm{ppm}$. Cada una de las determinaciones se llevó a cabo sobre una probeta con recubrimiento fotocatalítico en paralelo con otra probeta del mismo soporte sin recubrimiento.

El método utilizado para la determinación de la variación de concentración con el tiempo de exposición a la radiación fue el mismo que el indicado en los apartados anteriores.

Los resultados obtenidos indican que en todos los casos se obtiene una disminución de la concentración de AM con el tiempo de exposición a la radiación, siendo ésta más acusada que se produce por fotocatálisis lo que permite determinar la actividad del recubrimiento.

\section{CONCLUSIONES}

En el presente trabajo se ha puesto a punto un método para la determinación de la función fotocatalítica de recubrimientos aplicados sobre soporte cerámico.

El método ensayado consiste en la determinación de la variación de concentración que experimenta una disolución de azul de metileno cuando se expone a la radiación ultravioleta estando en contacto con el recubrimiento aplicado sobre el soporte cerámico. A partir de los resultados obtenidos ha sido posible obtener la cinética de la degradación que sufre el compuesto por la acción de la radiación ultravioleta y del 
recubrimiento.

El estudio de las variables relacionadas con los factores que intervienen en la realización del ensayo ha permitido establecer unas condiciones de ensayo estándar.

A partir de los ensayos realizados se concluye que el método empleado permite determinar la función fotocatalítica del recubrimiento obteniéndose distintos resultados según las características de la superficie del sustrato cerámico.

\section{AGRADECIMIENTOS}

Este trabajo se ha realizado con el apoyo del Fondo Europeo de Desarrollo Regional y del Instituto de la Pequeña y Mediana Industria de la Generalitat Valenciana (IMPIVA).

\section{BIBLIOGRAFÍA}

1. M.A. Fox, M. T. Dulay. «Heterogeneous Photocatalysis». Chem. Rev. 93, 341-357 (1993)

2. R. Benedix, F. Dehn, J. Quaas, M. Orgass. «Application of titanium dioxide photocatalysis to create self-cleaning building materials». Lacer 5, 157-168 (2000).

3. C. Ararat, J.A. Varela, J.E. Rodríguez-Páez., Uso de métodos químicos para obtener polvos cerámicos del sistema (Sn, Ti)O ${ }_{2}$, Bol. Soc. Esp. Ceram. V., 44, 4, 215-222 (2005)

4. A. Wold. «Photocatalytic properties of $\mathrm{TiO}_{2}$ " Chem. Mater., 5, 280-283 (1993).
5. A. Moreno, Ceramic tiles: above and beyond traditional applications, Bol. Soc. Esp. Ceram. V., 45, 2, 59-64 (2006).

6. A. Fujishima, X.T. Zhang. «Titanium dioxide photocatalysis: present situation and future approaches». Comptes Rendus Chimie 9 [5-6] 750-760 (2006).

7. C.H. Kwon, H.M. Shin, J.H. Kim, W.S. Choi, K.H. Yoon. «Degradation of methylene blue via photocatalysis of titanium dioxide». Mater. Chem. Phys. 86 [1] 78-82 (2004)

8. A. Houas, H. Lachleb, M. Ksibi, E. Elaloui, C. Guillard, J.M. Herrmann. «Photocatalytic degradation pathway of methylene blue in water». App. Catal. B: Environ. 31, 145-157 (2001).

9. Y.M. Wang, S.W. Liu, Z.L. Xiu, X.B. Jiao, X. B. Cui, J. Pan. «Preparation and photocatalytic properties of silica gel-supported $\mathrm{TiO}_{2} »$. Mater. Lett. 60[7] 974-978 (2006).

10. C. Guillard, H. Lachheb, A Houas, M. Ksibi, E. Elaloui, J.M. Herrmann. «Influence of chemical structure of dyes, of $\mathrm{pH}$ and of inorganic salts on their photocatalytic degradation by $\mathrm{TiO}_{2}$ comparison of the efficiency of powder and supported $\mathrm{TiO}_{2} . »$ J. Photochem. Photobiol. A-Chem. 158, 27-36 (2003).

11. W.S. Kuo, P.H. Ho. «Solar photocatalytic decolorization of dyes in solution with $\mathrm{TiO}_{2}$ film». Dyes Pigments 71[3] 212-217 (2006).

12. S. Lakshmi, R. Renganathan, S. Fujita. «Study on $\mathrm{TiO}_{2}$ - mediated photocatalytic degradation of methylene blue». J. Photochem. Photobiol. A-Chem. 88, 163-167 (1995).

13. C. Dingwang, K.R. Ajay. "Photocatalytic kinetics of phenol and its derivatives over UV irradiated $\mathrm{TiO}_{2}$. ». App. Catal. B: Environ. 23, 143-157 (1999).

Recibido: 12.01 .07

Aceptado: 8.11 .07 